\title{
A two-parameter generalization of the complete elliptic integral of second kind
}

\author{
Victor Bârsan \\ Department of Theoretical Physics, NIPNE, \\ Str. Atomistilor no. 407, Bucharest-Magurele, Romania
}

December 13, 2018

\begin{abstract}
A two-parameter generalization of the complete elliptic integral of second kind is expressed in terms of the Appell function $F_{4}$. This function is further reduced to a quite simple bilinear form in the complete elliptic integrals $K$ and $E$. The physical applications are briefly mentioned.
\end{abstract}

The integral

$$
K\left(k_{1}, k_{2}\right)=\int_{0}^{\pi / 2} \int_{0}^{\pi / 2} \frac{d \theta d \varphi}{\sqrt{1-k_{1}^{2} \sin ^{2} \theta-k_{2}^{2} \sin ^{2} \varphi}}
$$

can be considered a two-parameter generalization of the complete elliptic integral of first kind $K$. Its dependence of the parameters $k_{1}, k_{2}$ is ([1, 531.07; [2], 3.1.5.16):

$$
K\left(k_{1}, k_{2}\right)=\frac{2}{1+k_{2}^{\prime}} K\left(k_{3}\right) K^{\prime}\left(k_{4}\right)
$$

where:

$$
k_{3}=\frac{k_{1}^{\prime}-\sqrt{1-k_{1}^{2}-k_{2}^{2}}}{1+k_{2}^{\prime}}, k_{4}=\frac{k_{1}^{\prime}+\sqrt{1-k_{1}^{2}-k_{2}^{2}}}{1+k_{2}^{\prime}}, k_{1}^{2}+k_{2}^{2}<1
$$

However, the integral

$$
E\left(k_{1}, k_{2}\right)=\int_{0}^{\pi / 2} \int_{0}^{\pi / 2} \sqrt{1-k_{1}^{2} \sin ^{2} \theta-k_{2}^{2} \sin ^{2} \varphi} d \theta d \varphi
$$

which can be considered a two-parameter generalization of the complete elliptic integral of second kind, has not a compact form, at the best of author's knowledge. This article aims to derive such a result for $E\left(k_{1}, k_{2}\right)$, a function which 
is interesting for applications in statistical physics: it is proportional to the statistical sum of a three dimensional system of weakly coupled Ginzburg-Landau chains (for the two dimensional variant of the problem, see ([3]).

The double integral (1) can be re-written as:

$$
E\left(k_{1}, k_{2}\right)=\frac{1}{4} \sqrt{1-\frac{k_{1}^{2}}{2}-\frac{k_{2}^{2}}{2}} \int_{0}^{\pi} \int_{0}^{\pi} \sqrt{1+A \cos x+B \cos y} d x d y
$$

with

$$
A=\frac{\frac{k_{1}^{2}}{2}}{1-\frac{k_{1}^{2}}{2}-\frac{k_{2}^{2}}{2}}, B=\frac{\frac{k_{2}^{2}}{2}}{1-\frac{k_{1}^{2}}{2}-\frac{k_{2}^{2}}{2}}
$$

Using the formula ([4] 2.617.5, 3.671.1):

$$
\int_{0}^{\pi} \sqrt{a+b \cos x} d x=2 \sqrt{a+b} E(k)
$$

where

$$
k^{2}=\frac{2 b}{a+b} ; \quad a, b>0
$$

we get:

$$
\int_{0}^{\pi} \sqrt{1+A \cos x+B \cos y} d x=2 \sqrt{1+A+B \cos y} E\left(\sqrt{\frac{2 A}{1+A+B \cos y}}\right)
$$

Let us put

$$
I=\int_{0}^{\pi} \int_{0}^{\pi} \sqrt{1+A \cos x+B \cos y} d x d y
$$

We have:

$$
I=2 \int_{0}^{\pi} \sqrt{1+A+B \cos y} E\left(\sqrt{\frac{2 A}{1+A+B \cos y}}\right) d y
$$

Changing the variable:

$$
z^{2}=\frac{2 A}{1+A+B \cos y}
$$

we get:

$$
I=\sqrt{2 A} z_{0} z_{\pi} \int_{z_{0}}^{z_{\pi}} \frac{E(z) d z}{z^{2} \sqrt{\left(z^{2}-z_{0}^{2}\right)\left(z_{\pi}^{2}-z^{2}\right)}}
$$

with 


$$
z_{0}^{2}=\frac{2 A}{1+A+B} ; \quad z_{\pi}^{2}=\frac{2 A}{1+A-B}
$$

Writing the elliptic integral $E$ as a hypergeometric series:

$$
E(z)=\frac{\pi}{2} F\left(-\frac{1}{2}, \frac{1}{2} ; 1 ; z^{2}\right)=\frac{\pi}{2} \sum_{n=0}^{\infty} \frac{\left(-\frac{1}{2}\right)_{n}\left(\frac{1}{2}\right)_{n}}{n !(1)_{n}} z^{2 n}
$$

the integral (13) becomes:

$$
I=2 \pi \sqrt{2 A} z_{0} z_{\pi} \sum_{n=0}^{\infty} \frac{\left(-\frac{1}{2}\right)_{n}\left(\frac{1}{2}\right)_{n}}{(n !)^{2}} \int_{z_{0}}^{z_{\pi}} \frac{z^{2 n-2} d z}{\sqrt{\left(z^{2}-z_{0}^{2}\right)\left(z_{\pi}^{2}-z^{2}\right)}}
$$

Adapting the formula ([1, 218.15)

$$
\int_{y}^{a} \frac{R\left(t^{2}\right) d t}{\sqrt{\left(a^{2}-t^{2}\right)\left(t^{2}-b^{2}\right)}}=g \int_{0}^{u_{1}} R\left(a^{2} \operatorname{dn}^{2} u\right) d u
$$

with the conditions

$$
a>y \geq b>0
$$

and the notations:

$$
k^{2}=\frac{a^{2}-b^{2}}{a^{2}} ; \quad g=\frac{1}{a} ; \quad \sin \psi=\sqrt{\frac{a^{2}-y^{2}}{a^{2}-b^{2}}}
$$

to the integral in (17), we must put:

$$
a=z_{\pi}, \quad y=b=z_{0}
$$

The values of parameters in (17) are:

$$
k^{2}=\frac{2 B}{1+A+B}, \quad g=\sqrt{\frac{1+A-B}{2 A}}, \quad \sin \psi=1
$$

so the integral is complete and takes the form:

$$
\int_{z_{0}}^{z_{\pi}} \frac{R\left(z^{2}\right) d z}{\sqrt{\left(z^{2}-z_{0}^{2}\right)\left(z_{\pi}^{2}-z^{2}\right)}}=\sqrt{\frac{1+A-B}{2 A}} \int_{0}^{K} R\left(\frac{2 A}{1+A-B} \operatorname{dn}^{2} u\right) d u
$$

But ([1] 810.00)

$$
P_{m-1 / 2}(x)=\frac{2}{\pi}\left(x+\sqrt{x^{2}-1}\right)^{m-1 / 2} \int_{0}^{K} \mathrm{dn}^{2 m} u d u
$$

with

$$
k^{2}=2\left[x \sqrt{x^{2}-1}+1-x^{2}\right]
$$


Inverting (24), we obtain:

$$
x^{2}=\frac{\left(1-\frac{k^{2}}{2}\right)^{2}}{1-k^{2}}
$$

or, with (21) and (6),

$$
x^{2}=\frac{(1+A)^{2}}{(1+A)^{2}-B^{2}}
$$

and (23) gives:

$$
\int_{0}^{K} \operatorname{dn}^{2 m} u d u=\frac{\pi}{2}\left(\frac{1+A-B}{1+A+B}\right)^{\frac{1}{2}\left(m-\frac{1}{2}\right)} P_{m-\frac{1}{2}}\left(\frac{1+A}{\sqrt{(1+A)^{2}-B^{2}}}\right)
$$

So, finally,

$$
\int_{z_{0}}^{z_{\pi}} \frac{z^{2 n-2} d z}{\sqrt{\left(z^{2}-z_{0}^{2}\right)\left(z_{\pi}^{2}-z^{2}\right)}}=\frac{\pi}{2}\left(\frac{2 A}{\sqrt{(1+A)^{2}-B^{2}}}\right)^{n-\frac{3}{2}} P_{n-\frac{3}{2}}\left(\frac{1+A}{\sqrt{(1+A)^{2}-B^{2}}}\right)
$$

and

$$
I=\pi^{2} \sqrt{2 A} z_{0} z_{\pi} \sum_{n=0}^{\infty} \frac{\left(-\frac{1}{2}\right)_{n}\left(\frac{1}{2}\right)_{n}}{(n !)^{2}} t^{n-\frac{3}{2}} P_{n-\frac{3}{2}}(x)
$$

with the notations:

$$
t=\frac{2 A}{\sqrt{(1+A)^{2}-B^{2}}}, \quad x=\frac{1+A}{\sqrt{(1+A)^{2}-B^{2}}}
$$

Observing that:

$$
z_{0} z_{\pi}=t
$$

eq. (28) can be written as:

$$
I=\pi^{2} \sqrt{2 A} \sum_{n=0}^{\infty} \frac{\left(-\frac{1}{2}\right)_{n}\left(\frac{1}{2}\right)_{n}}{n !(1)_{n}} t^{n-\frac{1}{2}} P_{n-\frac{3}{2}}(x)
$$

Writing

$$
\left(\frac{1}{2}\right)_{k}=-2\left(k-\frac{1}{2}\right)\left(-\frac{1}{2}\right)_{k}
$$

the previous expression takes the form: 


$$
I=-2 \pi^{2} \sqrt{2 A} \sum_{n=0}^{\infty}\left[\frac{\left(-\frac{1}{2}\right)_{n}}{n !}\right]^{2}\left(n-\frac{1}{2}\right) t^{n-\frac{1}{2}} P_{n-\frac{3}{2}}(x)
$$

The sum (29) can be put in a compact form using the formula ([5] 6.5.1.18 for $\mu=0)$

$\sum_{k=0}^{\infty} \frac{(1+\nu)_{k}(1+\nu)_{k}}{k !(1)_{k}} t^{k} P_{k+\nu}(x)=\left(\frac{x+1}{2}\right)^{-\nu-1} F_{4}\left(1+\nu, 1+\nu ; 1,1 ; \frac{2 t}{1+x}, \frac{x-1}{x+1}\right)$

With $\nu=-\frac{3}{2}$ :

$$
\begin{aligned}
& \sum_{k=0}^{\infty} \frac{\left(\left(-\frac{1}{2}\right)_{k}\right)^{2}}{(k !)^{2}} t^{k} P_{k-3 / 2}(x)=\left(\frac{x+1}{2}\right)^{1 / 2} F_{4}\left(-\frac{1}{2},-\frac{1}{2} ; 1,1 ; \frac{2 t}{1+x}, \frac{x-1}{x+1}\right) \\
& I=-2 \pi^{2} \sqrt{2 A}\left(\frac{x+1}{2}\right)^{1 / 2} t \frac{\partial}{\partial t} t^{-1 / 2} F_{4}\left(-\frac{1}{2},-\frac{1}{2} ; 1,1 ; \frac{2 t}{1+x}, \frac{x-1}{x+1}\right)
\end{aligned}
$$

where the function $\frac{\partial}{\partial t} F_{4}$ must be taken in the point $(t, x)$ defined by (30). The arguments of the Appell function $F_{4}$ in (34) can be expressed in terms of the parameters $k_{1}, k_{2}$ using the relations:

$$
\begin{gathered}
t=\frac{k_{1}^{2}}{k_{2}^{\prime}}, \quad x=\frac{1}{2} \frac{1+k_{2}^{\prime 2}}{k_{2}^{\prime}} \\
\frac{2 t}{1+x}=\frac{4 k_{1}^{2}}{\left(1+k_{2}^{\prime}\right)^{2}} ; \quad \frac{x-1}{x+1}=\frac{\left(1-k_{2}^{\prime}\right)^{2}}{\left(1+k_{2}^{\prime}\right)^{2}}
\end{gathered}
$$

The parameters of the Appell function are very particular, and the function can be "reduced", using the formula ([6, 8.4.14):

$$
\begin{gathered}
F_{4}\left[a, b ; c, c^{\prime} ; x(1-y), y(1-x)\right]=\sum_{r=0}^{\infty}(a)_{r}(b)_{r} \frac{\left(1+a+b-c-c^{\prime}\right)}{r !(c)\left(c^{\prime}\right)} x^{r} y^{r} . \\
\cdot F(a+r, b+r ; c+r ; x) F\left(a+r, b+r ; c^{\prime}+r ; y\right)
\end{gathered}
$$

with $F$ - the Gaussian hypergeometric function.

In our case,

$$
a=b=-\frac{1}{2}, c=c^{\prime}=1
$$

so: 


$$
\begin{aligned}
& F_{4}\left[-\frac{1}{2},-\frac{1}{2} ; 1,1 ; u(1-v), v(1-u)\right]=F\left(-\frac{1}{2},-\frac{1}{2} ; 1 ; u\right) \cdot F\left(-\frac{1}{2},-\frac{1}{2} ; 1 ; v\right)- \\
& -\frac{1}{2} u v\left(\frac{1}{2}, \frac{1}{2} ; 2 ; u\right) \cdot F\left(\frac{1}{2}, \frac{1}{2} ; 2 ; v\right)+\frac{1}{16} u^{2} v^{2} \cdot F\left(\frac{3}{2}, \frac{3}{2} ; 3 ; u\right) \cdot F\left(\frac{3}{2}, \frac{3}{2} ; 3 ; v\right)
\end{aligned}
$$

In order to apply (37), we have to solve the system:

$$
u(1-v)=\frac{2 t}{x+1}, \quad v(1-u)=\frac{x-1}{x+1}
$$

It has the solution:

$$
v=\frac{x-t \pm \sqrt{1-2 t x+t^{2}}}{x+1}, \quad u=\frac{1+t \pm \sqrt{1-2 t x+t^{2}}}{x+1}
$$

or

$$
\begin{gathered}
u=2 \frac{k_{1}^{2}+k_{2}^{\prime} \pm k_{1}^{\prime} \sqrt{1-k_{1}^{2}-k_{2}^{2}}}{\left(1+k_{2}^{\prime}\right)^{2}} ; \quad v=\frac{1-2 k_{1}^{2}+k_{2}^{\prime 2} \pm 2 k_{1}^{\prime} \sqrt{1-k_{1}^{2}-k_{2}^{2}}}{\left(1+k_{2}^{\prime}\right)^{2}} \\
\sqrt{1-2 t x+t^{2}}=\sqrt{1-k_{1}^{2}-k_{2}^{2}} \frac{k_{1}^{\prime}}{k_{2}^{\prime}}=k^{\prime} \frac{k_{1}^{\prime}}{k_{2}^{\prime}}
\end{gathered}
$$

Also, using the formula:

$$
\sqrt{A \pm \sqrt{B}}=\sqrt{\frac{A+m}{2}} \pm \sqrt{\frac{A-m}{2}}
$$

we get:

$$
\begin{gathered}
\sqrt{u}=\frac{1}{1+k_{2}^{\prime}}\left(\sqrt{\left(1+k_{1}\right)\left(k_{2}^{\prime}+k_{1}\right)} \pm \sqrt{\left(1-k_{1}\right)\left(k_{2}^{\prime}-k_{1}\right)}\right) \\
\sqrt{v}=\frac{1}{1+k_{2}^{\prime}}\left(k_{1}^{\prime} \pm \sqrt{1-k_{1}^{2}-k_{2}^{2}}\right)
\end{gathered}
$$

If we put:

$$
k^{2}=k_{1}^{2}+k_{2}^{2} ; \quad k^{\prime 2}=1-k^{2}=1-1-k_{1}^{2}-k_{2}^{2}
$$

we can write $u, v$ as:

$$
u=2 \frac{k_{1}^{2}+k_{2}^{\prime} \pm k_{1}^{\prime} k^{\prime}}{\left(1+k_{2}^{\prime}\right)^{2}} ; \quad v=\frac{1-2 k_{1}^{2}+k_{2}^{\prime 2} \pm 2 k_{1}^{\prime} k^{\prime}}{\left(1+k_{2}^{\prime}\right)^{2}}
$$

Also, 


$$
\begin{aligned}
& \frac{\partial}{\partial t} u=\dot{u}=\frac{1}{\left(1+k_{2}^{\prime}\right)^{2}} \frac{k_{2}^{\prime}}{k^{\prime} k_{1}^{\prime}}\left[2 k^{\prime} k_{1}^{\prime} \mp\left(2 k^{\prime}+k_{2}^{2}\right)\right] \\
& \frac{\partial}{\partial t} v=\dot{v}=\frac{1}{\left(1+k_{2}^{\prime}\right)^{2}} \frac{k_{2}^{\prime}}{k^{\prime} k_{1}^{\prime}}\left[-2 k^{\prime} k_{1}^{\prime} \mp\left(2 k^{\prime}+k_{2}^{2}\right)\right]
\end{aligned}
$$

Both roots $( \pm)$ satisfy the relation:

$$
0<u, v<1
$$

which results, in fact, from (40). By direct substitution in the formula (39), for $k_{1}, k_{2} \rightarrow 0$, we can check that only the roots $u_{-}, v_{-}$must be used and finally we obtain:

$$
\begin{gathered}
F_{4}\left(-\frac{1}{2},-\frac{1}{2} ; 1,1 ; \frac{2 t}{1+x}, \frac{x-1}{x+1}\right)= \\
=F\left(-\frac{1}{2},-\frac{1}{2} ; 1 ; u\right) F\left(-\frac{1}{2},-\frac{1}{2} ; 1 ; v\right)-\frac{1}{2} u v F\left(\frac{1}{2}, \frac{1}{2} ; 2 ; u\right) F\left(\frac{1}{2}, \frac{1}{2} ; 2 ; v\right)+ \\
+\frac{1}{16} u^{2} v^{2} F\left(\frac{3}{2}, \frac{3}{2} ; 3 ; u\right) F\left(\frac{3}{2}, \frac{3}{2} ; 3 ; v\right)
\end{gathered}
$$

where

$$
u=u(x, t)=u\left(k_{1}, k_{2}\right)=u_{-}, \quad v=v(x, t)=v\left(k_{1}, k_{2}\right)=v_{-}
$$

are defined by (41, 42).

Let us express the r.h.s. of the formula (52) in terms of elliptic integrals. According to Slater (1.4.1.1):

$$
\frac{d}{d z} F(a, b ; c ; z)=\frac{a b}{c} F(a+1, b+1 ; c+1 ; z)
$$

Consequently,

$$
\begin{array}{r}
F\left(\frac{1}{2}, \frac{1}{2} ; 2 ; z\right)=4 \frac{d}{d z} F\left(-\frac{1}{2},-\frac{1}{2} ; 1 ; z\right)=4 F^{\prime}\left(-\frac{1}{2},-\frac{1}{2} ; 1 ; z\right) \\
F\left(\frac{3}{2}, \frac{3}{2} ; 3 ; z\right)=32 \frac{d^{2}}{d z^{2}} F\left(-\frac{1}{2},-\frac{1}{2} ; 1 ; z\right)=32 F^{\prime \prime}\left(-\frac{1}{2},-\frac{1}{2} ; 1 ; z\right)
\end{array}
$$

and

$$
F_{4}\left(-\frac{1}{2},-\frac{1}{2} ; 1,1 ; \frac{2 t}{1+x}, \frac{x-1}{x+1}\right)=
$$




$$
\begin{aligned}
& =F\left(-\frac{1}{2},-\frac{1}{2} ; 1 ; u\right) F\left(-\frac{1}{2},-\frac{1}{2} ; 1 ; v\right)-8 u v F^{\prime}\left(-\frac{1}{2},-\frac{1}{2} ; 1 ; u\right) F^{\prime}\left(-\frac{1}{2},-\frac{1}{2} ; 1 ; v\right)+ \\
& +64 u^{2} v^{2} F^{\prime \prime}\left(-\frac{1}{2},-\frac{1}{2} ; 1 ; u\right) F^{\prime \prime}\left(-\frac{1}{2},-\frac{1}{2} ; 1 ; v\right)
\end{aligned}
$$

It is easy to show that:

$$
F\left(-\frac{1}{2},-\frac{1}{2} ; 1 ; z\right)=4 z^{1 / 2}(1-z)^{2} \frac{d}{d z} z \frac{d}{d z} z^{1 / 2} F\left(\frac{1}{2}, \frac{1}{2} ; 1 ; z\right)
$$

Also,

$$
F\left(\frac{1}{2}, \frac{1}{2} ; 1 ; z\right)=\frac{2}{\pi} K(\sqrt{z})
$$

With the formulae of the derivatives of the complete elliptic integrals (see for instance ([1] 710.00, 710.02)), we find:

$$
F\left(-\frac{1}{2},-\frac{1}{2} ; 1 ; z\right)=\frac{2}{\pi}[2 E(\sqrt{z})-(1-z) K(\sqrt{z})]
$$

Similarly,

$$
\begin{gathered}
F^{\prime}\left(-\frac{1}{2},-\frac{1}{2} ; 1 ; z\right)=\frac{1}{\pi z}[E(\sqrt{z})-(1-z) K(\sqrt{z})] \\
F^{\prime \prime}\left(-\frac{1}{2},-\frac{1}{2} ; 1 ; z\right)=\frac{1}{2 \pi z^{2}}[(2-z) K(\sqrt{z})-2 E(\sqrt{z})]
\end{gathered}
$$

Finally, we obtain:

$$
\begin{gathered}
F_{4}\left(-\frac{1}{2},-\frac{1}{2} ; 1,1 ; \frac{2 t}{1+x}, \frac{x-1}{x+1}\right)= \\
=\frac{4}{\pi^{2}}\{66 E(\sqrt{u}) E(\sqrt{v})-32(2-v) E(\sqrt{u}) K(\sqrt{v})-32(2-u) E(\sqrt{v}) K(\sqrt{u}) \\
+[63-31(u+v)+15 u v] K(\sqrt{u}) K(\sqrt{v})\} .
\end{gathered}
$$

Also,

$$
\begin{aligned}
& \frac{\partial}{\partial t} E(\sqrt{u})=\frac{1}{2 u}[E(\sqrt{u})-K(\sqrt{u})] \dot{u} \\
& \frac{\partial}{\partial t} K(\sqrt{u})=\frac{1}{2 u}\left[\frac{E(\sqrt{u})}{1-u}-K(\sqrt{u})\right] \dot{u}
\end{aligned}
$$

In spite of the reducibility of the Appell function $F_{4}$, the most compact formula for $E\left(k_{1}, k_{2}\right)$ is: 


$$
E\left(k_{1}, k_{2}\right)=-\frac{\pi^{2}}{2} k_{1}\left(\frac{x+1}{2}\right)^{1 / 2} t \frac{\partial}{\partial t}\left[t^{-1 / 2} F_{4}\left(-\frac{1}{2},-\frac{1}{2} ; 1,1 ; \frac{2 t}{1+x}, \frac{x-1}{x+1}\right)\right]
$$

where the r.h.s. must be taken in the point $(t, x)$ defined by (30)

For the applications in statistical mechanics, the particular case $k_{1}=k_{2}$ is important. The arguments of the Appell function simplifies, in the sense that:

$$
F_{4}\left(-\frac{1}{2},-\frac{1}{2} ; 1,1 ; \frac{2 t}{1+x}, \frac{x-1}{x+1}\right)=F_{4}\left(-\frac{1}{2},-\frac{1}{2} ; 1,1 ; 4 \xi, \xi^{2}\right)
$$

with

$$
\xi=\frac{1+A-\sqrt{1+2 A}}{A}
$$

The convergence of the double series defined by the Appell functions is assured if $(7])$

$$
2 \sqrt{\xi}+\xi<1
$$

or

$$
A<\frac{1}{2}
$$

In terms of the parameters $k_{1}, k_{2}$,

$$
k_{1}=k_{2}<\frac{1}{\sqrt{2}}
$$

The formulae (65), (66) allow us to find the exact thermodynamics of a three dimensional anisotropic physical system, namely a regular array of parallel Ginzburg-Landau chains.

The author is indebted to Prof. D. Grecu and Prof. N. Grama for useful discussions and suggestions.

\section{References}

[1] Byrde P. F. and Friedman M. D., Handbook of elliptic integrals for engineers and physicists, Springer Verlag, Berlin, Göttingen, Heidelberg, 2nd edition, 1971

[2] Prudnikov A. P., Brychkov Yu. A. and Marichev O. I., Integrals and series. Elementary functions, Nauka, Moscow, 1981 (in Russian)

[3] Bârsan V. J. Phys. Cond. Mat. 189273 (2006) 
[4] Gradstein I. S. and Ryzhik I. M., Tables of integrals, series and products, Academic Press, New York, London, 1980

[5] Prudnikov A. P., Brychkov Yu. A. and Marichev O. I., Integrals and series. Complementary chapters, Nauka, Moscow, 1981 (in Russian)

[6] Slater L. J., Generalized hypergeometric functions, Cambridge University Press, 1966

[7] Bayley W. N. Generalized hypergeometric series, Cambridge University Press, 1964 